Mater. Res. Soc. Symp. Proc. Vol. 1794 (C) 2015 Materials Research Society

DOI: 10.1557/opl.2015.638

\title{
A Molecular Picture for the Thermo-Reversibility of Gels Formed by Isophthalic Acid- Ended Telechelic Polymers
}

\author{
Boyu Li, Joey Kim and Julie Kornfield \\ Chemistry and Chemical Engineering, California Institute of Technology, 1200 E. California \\ Blvd. Pasadena, CA 91125, U.S.A.
}

\begin{abstract}
We demonstrate that isophthalic acid-ended telechelic poly(1,5-cyclooctadiene)s (APCODs) form thermo-reversible gels in non-polar solvent with a unique molecular mechanism for their thermo-reversibility. Like other associative telechelic polymers, A-PCODs form "flower-like" micelles at low concentration and form gels through bridging at higher concentration which exhibit linear viscoelasticity. However, unlike the widely studied hydrophobically end-capped PEOs, APCODs show clear thermo-reversibility in viscosity and dynamic modulus around $30^{\circ} \mathrm{C}$ due to the hydrogen-bonding end groups. In addition, they differ from other reported thermo-reversible gelators (eg. Pluronics, PNIPAm containing block copolymers, etc.): neither the end group nor the backbone in the present system has a critical solution temperature within the measured temperature range $\left(0^{\circ} \mathrm{C}\right.$ to $\left.60^{\circ} \mathrm{C}\right)$, indicating that the present system has a unique mechanism for its thermoreversibility. To obtain a molecular picture of the mechanism, rheology and small angle neutron scattering (SANS) studies were implemented. Topological changes above the transition temperature $\left(30^{\circ} \mathrm{C}\right)$ were observed in both oscillatory rheology and SANS. SANS reveals that the size of clusters, which are formed by interacting micelles, depends highly on temperature $(\mathrm{T})$ but independent of polymer concentration. These results cannot be explained by current theories on associative telechelic polymers which assume constant and large aggregation number of end groups at all temperatures and concentrations. We hypothesize that the temperature-sensitive solgel transition is due to a decrease in aggregation number for $T$ above the critical temperature in our system, and this temperature-dependence of aggregation number is further determined by the chemical structure and hydrogen-bonding property of isophthalic acid ends.
\end{abstract}

\section{INTRODUCTION}

Associative telechelic polymers, especially hydrophobically modified poly(ethylene oxides) (PEOs), have been widely studied and applied to solutions to control rheological properties such as viscosity and gelation and shear-thinning and thickening behavior [1]. These polymers form flower-like micelles at low concentrations, begins to bridge as concentration is increased, and eventually form soft gels [1].

Recently, the linear viscoelasticity of the resulting gels (or thick solutions) has attracted great attention. Time-temperature superposition (TTS) could be applied to their dynamic modulus and the resulting master curve could be fitted by the Maxwell model with a single relaxation time corresponding to the dissociation rate of end groups from the core of the micelle [2]. Both zero- 
shear viscosity and relaxation time follow an Arrhenius-type temperature dependence of similar activation energy. This result, in conjunction with the success of TTS, demonstrates that the highfrequency modulus is independent of temperature, in which we can infer no topological change takes place within the measured temperature range.

Many theories have been developed for associative telechelic polymers and can be divided into two categories. Transient network theory, from the kinetic theory of rubber elasticity, suggests that the elastic modulus is proportional to the number of elastically active bridges [2-4]. Another branch of theory, which we will refer to as 'interacting micelle theory', postulates that the dynamic of the system is determined by the interaction between micelles: micelles attract due to the entropy gain from the exchange of end blocks between cores of flower-like micelles $[5,6]$. Both theories assume that the aggregation number of end groups within a micelle core doesn't change with temperature, which is in agreement with the experimental data showing no temperature-dependent topological change.

In contrast to hydrophobically modified PEOs, which are not thermo-sensitive due to the strong hydrophobic effect of the end groups, block copolymers with end blocks having critical solution temperatures have been widely studied for their thermo-reversibility. End blocks experience an abrupt change in their solubility around the critical temperature, which leads to a temperature-dependent aggregation number and topology.

In the present work, we show that isophthalic acid-ended telechelic polycyclooctadienes (A-PCODs, Scheme 1 right) form thermo-reversible viscoelastic gels in non-polar solvent with a unique molecular mechanism for their thermo-reversibility - a mechanism not covered by current theories and not due to critical solution temperatures.

\section{EXPERIMENTAL DETAILS}

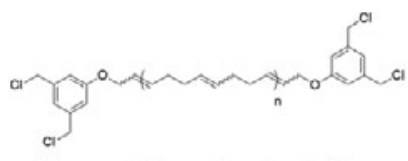

Non-associative polycyclooctadiene (NA-PCOD)

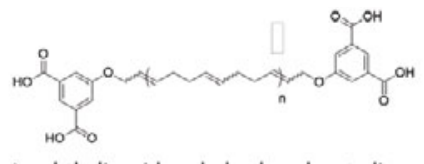

Isophthalic acid-ended polycyclooctadiene (A-PCOD)

Scheme 1: Structures for matched sets of non-associative and self-associative telechelic polvmers.

Two types of end-groups ("NA": non-associative, "A": isophthalic acid) were installed at the ends of our telechelic poly(1,5-cyclooctadiene)s (PCODs) to produce precisely matched sets of non-associative and self-associative polymers (Scheme 1). Decalin (cis/trans mix) was chosen as the solvent 1) for its low polarity which facilitates the hydrogen bonding between acid ends, and 2) to provide athermal solvent conditions for the PCOD backbone. In an athermal solvent, the solvent quality is independent of temperature thus the changes in the system with temperature can be solely attributed to end-group interactions. 
Rheology. Polymers were dissolved in decalin using a shaker at room temperature. Shear-flow rheology data were obtained from $0^{\circ} \mathrm{C}$ to $60^{\circ} \mathrm{C}$ with stress-controlled rheometer TA AR1000, equipped with a cone-plate geometry (angle $1^{\circ}$, diameter $60 \mathrm{~mm}$ ) with shear rate range depending on the temperature and concentration (as shown in Figure 2A). The viscosities shown in Figure $1 \mathrm{~A}$ are averages of shear independent data points. In addition, oscillatory rheology tests were implemented with stress-controlled rheometer TA AR1000, equipped with a cone-plate geometry (angle $1^{\circ}$, diameter $60 \mathrm{~mm}$ ) at $20 \%$ strain from $0{ }^{\circ} \mathrm{C}$ to $60^{\circ} \mathrm{C}$.

Small angle neutron scattering (SANS). Decalin- $d_{18}$ (cis/trans mix) solutions of polymers (nonassociative polycyclooctadiene and isophthalic acid-ended polycyclooctadiene) were prepared by weighing out polymer on a Mettler precision balance $( \pm 0.01 \mathrm{mg})$ into new glass scintillation vials with PTFE lined caps and subsequently adding the appropriate amount of solvent using a precision syringe $( \pm 1 \%)$. These were placed on a wrist action shaker at room temperature overnight.

SANS patterns were obtained at Oak Ridge National Laboratory (ORNL) on beamline CG-2 at the High Flux Isotope Reactor (HFIR). Samples were placed in Hellma quartz cylindrical cells with $5 \mathrm{~mm}$ path length. Scattering experiments were conducted from $0^{\circ} \mathrm{C}$ to $60^{\circ} \mathrm{C}$ with Peltier temperature control. At $0{ }^{\circ} \mathrm{C}$, sample holders were placed under $\mathrm{N}_{2}$ flow to avoid moisture condensation on Hellma cells. Two-dimensional scattering patterns were taken for each sample using three detector distances $(1 \mathrm{~m}, 10 \mathrm{~m}$ and $18.5 \mathrm{~m})$. The overall scattering vector ranges were $0.0015 \AA^{-1}<\mathrm{q}<0.4 \AA^{-1}$.

\section{RESULTS AND DISCUSSION}
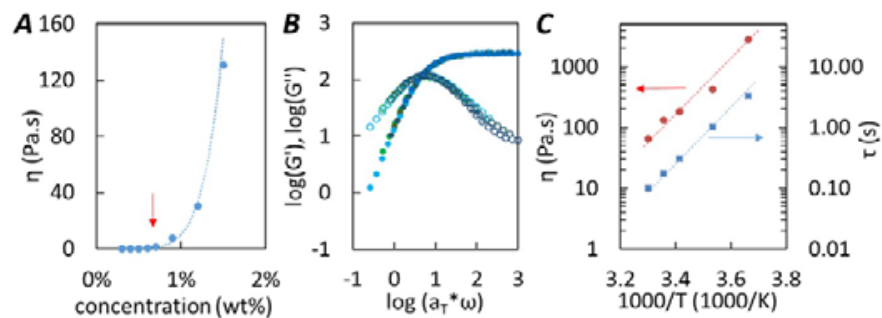

Figure 1: A. Shear rheology data of decalin solutions of A-PCOD $50 \mathrm{~kg} / \mathrm{mol}$. B. Time-temperature superposition for $1.5 \mathrm{wt} \%$ decalin solution of $50 \mathrm{~kg} / \mathrm{mol} \mathrm{A}-\mathrm{PCOD}$ solution $\left(0^{\circ} \mathrm{C}\right.$ to $30^{\circ} \mathrm{C}$, with $25^{\circ} \mathrm{C}$ as reference temperature). C. Arrhenius plot of viscosity and relaxation time for $1.5 \mathrm{wt} \%$ decalin solution of $50 \mathrm{~kg} / \mathrm{mol} \mathrm{A-PCOD}$ solution.

$50 \mathrm{~kg} / \mathrm{mol}$ isophthalic acid-ended polycyclooctadiene (A-PCOD) clearly shows some typical rheological characteristics as the widely studied associative telechelic polymers. Shear rheology at $25{ }^{\circ} \mathrm{C}$ shows a sharp upturn in viscosity at roughly $0.6 \% \mathrm{wt}$ (Figure 1A) which is indicative of the overlap concentration of $50 \mathrm{~kg} / \mathrm{mol}$ PCOD without associative ends. Oscillatory rheology demonstrates that time-temperature superposition could be applied to dynamic modulus 
curves obtained for temperatures $(\mathrm{T})$ below $30{ }^{\circ} \mathrm{C}$ without vertical shift (Figure 1B). The master curve could be fitted with Maxwell model featuring one relaxation time- scale of pulling a single end out of the micelle core. Below $30^{\circ} \mathrm{C}$, both the zero-shear viscosity and the relaxation time follow an Arrhenius-type temperature dependence with similar activation energies, indicating the absence of topological change within this temperature range $\left(0^{\circ} \mathrm{C}\right.$ to $30^{\circ} \mathrm{C}$, Figure $\left.1 \mathrm{C}\right)$.

Although typical behaviors of telechelic polymers are observed, A-PCOD system also shows some unique features. First, despite interacting micelle theory predicting phase separation for hydrophobically modified PEOs, there was no phase separation in the present system even in dilute solutions $(0.3 \% \mathrm{wt})$. Second, unlike hydrophobically modified PEOs, the gelation of APCOD is thermo-reversible - $2 \%$ wt A-PCOD solution in decalin gels at room temperature and liquefies when heated. In rheology, A-PCOD solutions show significant reduction in viscosity with heating above the transition temperature $30{ }^{\circ} \mathrm{C}$ (Figure 2A). Accordingly, oscillatory rheology revealed the dynamic modulus curves obtained above $30{ }^{\circ} \mathrm{C}$ could not fit in the master curves (Figure 2B), indicating the presence of topological changes associating with the thermoreversibility.
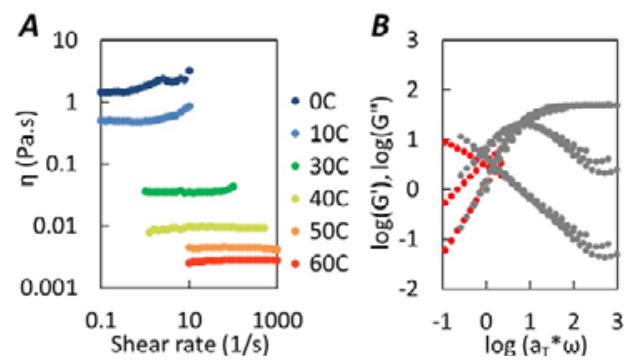

Figure 2: A. Viscosities of $0.6 \%$ wt APCOD solution at different temperatures. B. Master curves of $0.9 \%$ wt A-PCOD solution (G', G" and $\tan \delta, 0{ }^{\circ} \mathrm{C}$ to $30^{\circ} \mathrm{C}$ with $25^{\circ} \mathrm{C}$ as reference $\mathrm{T}$, grey) and the curves obtained at $50{ }^{\circ} \mathrm{C}$ (red).

SANS experiments were designed to obtain a molecular insight for the thermo-sensitive sol-gel transition. Measurements with A-PCOD in d-decalin showed morphological changes above a critical temperature (around $30^{\circ} \mathrm{C}$, Figure $3 \mathrm{~A}$ yellow and red), but showed minute changes below $30{ }^{\circ} \mathrm{C}$ (Figure $3 \mathrm{~A}$, green and two blues). This temperature dependence holds true for both $0.6 \mathrm{wt} \%$ and $0.3 \mathrm{wt} \%$. It is important to note that although the viscosities of $0.6 \mathrm{wt} \%$ solution are dramatically higher than those of $0.3 \mathrm{wt} \%$ solution (Figure 3B top, due to the onset of network formation at $0.6 \mathrm{wt} \%$ ), the $0.6 \mathrm{wt} \%$ and $0.3 \mathrm{wt} \%$ solutions give nearly identical SANS curves (intensities normalized by concentration) at each temperature (Figure 3B bottom).

The abovementioned findings could not be explained by neither transient network theory nor interacting micelle theory. Transient network theory assumes that elastic modulus is only controlled by the number of bridging chains in the system and the fraction of bridging chains is larger at higher concentration [2], which would result in larger clusters with increasing concentration $(0.3 \mathrm{wt} \%$ vs. $0.6 \mathrm{wt} \%)$. However, the identical SANS curves for $0.3 \mathrm{wt} \%$ and $0.6 \mathrm{wt} \%$ solutions suggest that the cluster size of our system is independent of concentration and the transient network theory is not applicable to the sol-gel transition of A-PCOD solutions. In addition, the interacting micelle theory assumes that micelles have an entropic attraction to each other: 
isolated micelles consist solely of loops while micelle interaction leads to an increase in conformations due to the exchange of end blocks between cores of micelles. The micelle-micelle interaction is strong enough for the solution to phase separate $[5,6]$. This interaction energy only depends on the aggregation number of end groups, $p$, with larger $p$ values indicating stronger interactions and $p$ is invariant in T [5,6]. Our system differs from the interacting micelle theory in two ways: 1) lack of phase separation and 2) SANS shows the cluster size decreases for T above $30^{\circ} \mathrm{C}$.
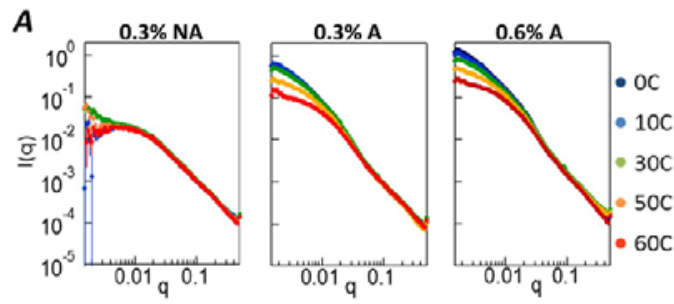

Figure 3: A. SANS of 50 $\mathrm{kg} / \mathrm{mol}$ NA-PCOD and APCOD in d-decalin at $0.6 \mathrm{wt} \%$ and $0.3 \mathrm{wt} \%$. The backbone (NA-PCOD) shows no conformation change with $\mathrm{T}$.
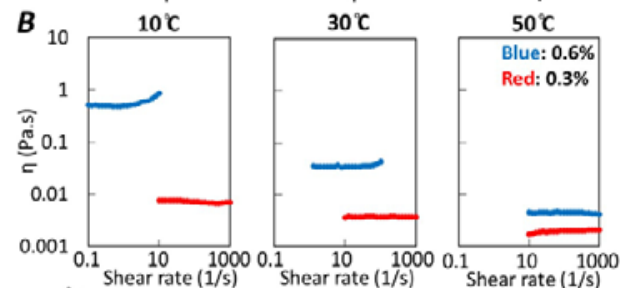

B. Viscosities and SANS of 50 $\mathrm{kg} / \mathrm{mol}$ A-PCOD solutions $(0.6 \mathrm{wt} \%$ and $0.3 \mathrm{wt} \%)$ at $10^{\circ} \mathrm{C}$, $30^{\circ} \mathrm{C}$ and $50^{\circ} \mathrm{C}$. The units for $\mathrm{q}$ is $\AA^{-1}$.
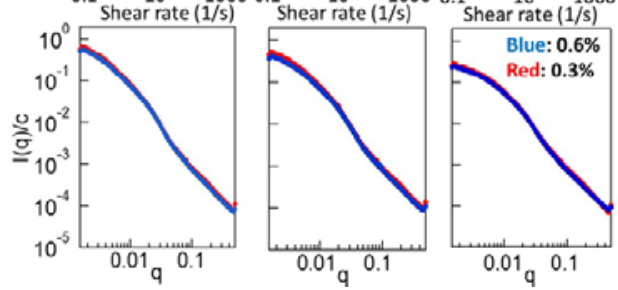

We hypothesize that the temperature-sensitive sol-gel transition is due to a decreasing aggregation number with increasing $\mathrm{T}$ above $30^{\circ} \mathrm{C}$. We assume that SANS only depicts the size of the clusters formed by micelle-micelle aggregation (both $0.3 \%$ and $0.6 \%$ ) even though these clusters themselves form a network at $0.6 \%$. As shown in Figure 3, the cluster size is dependent on temperature (Figure 3A), but not on concentration (Figure 3B bottom). According to the interacting micelle theory $[5,6]$ the cluster size depends on the micelle-micelle interaction energy, and hence, the aggregation number of end groups, thus a decrease in cluster size suggests a decrease in aggregation number with increasing $\mathrm{T}$ above $30^{\circ} \mathrm{C}$.

The temperature dependence of aggregation number may be explained by the chemical structure and hydrogen-bonding properties of isophthalic acid: one isophthalic acid has two 
carboxylic acid groups, separated by $120^{\circ}$, which could each form a dimer. We show here some hypothetical average aggregation numbers. At high $\mathrm{T}$, larger fraction of dangling acid groups may be tolerated, corresponding to low aggregation number $p$ (Figure $4, p=2$ ). With decreasing $\mathrm{T}$, the fraction of dangling acids decreases, leading to larger values of $p$ (Figure $4, p=4$ ) until all hydrogen bonds are satisfied and no dangling acids are left (Figure $4, p=6$ ). This hypothesis is in agreement to our findings that the cluster size becomes temperature independent below $30^{\circ} \mathrm{C}$. It is important to note the hypothesized interaction of isophthalic acid in

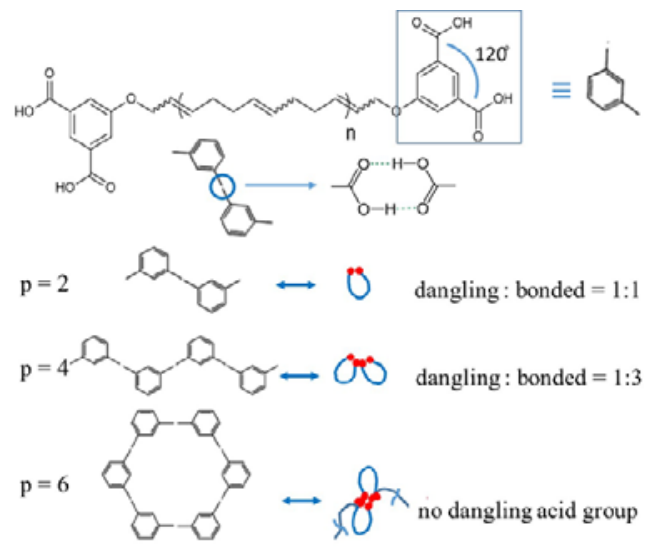

Figure 4: Hypothesis for the mechanism of the temperature dependence of aggregation number ( $\mathrm{p}$ )

Figure 4 does not consider the presence of the polymer backbone and Figure 4 is a $2 \mathrm{D}$ representation of idealized structures. However, the entropic nature of polymers favor a $3 \mathrm{D}$ structure of the end-group aggregates, which may involve some distortions of the conjugation and hydrogen bonds of structures shown.

\section{CONCLUSIONS}

We demonstrate that isophthalic acid-ended telechelic polycyclooctadienes (A-PCODs) form thermo-reversible viscoelastic gels in non-polar solvent. The mechanism for their thermoreversibility is postulated to be a decreasing aggregation number for $\mathrm{T}$ above the critical temperature $\left(30^{\circ} \mathrm{C}\right)$. This mechanism is not covered by current theories and, also, is not due to any critical solution temperature as in the case of other widely studied thermo-reversible gelators. The temperature dependence of aggregation numbers could be due to the chemical structure and the directionality of hydrogen bonding of isophthalic acid end groups. Future simulation study would be implemented to test our hypothesis.

\section{REFERENCES}

1. C. Chassenieux, T. Nicolai \& L. Benyahia, Current Opinion in Colloid \& Interface Science 16, 18 (2011).

2. T. Annable, R. Buscall, R. Ettelaie, \& D. Whittlestone, Journal of Rheology 37, 695 (1993).

3. M. S. Green \& A. V. Tobolsky, the Journal of Chemical Physics 14, 80 (1946).

4. F. Tanaka \& S. F. Edwards, Macromolecules 25, 1516 (1992).

5. A.N. Semenov, J.-F. Joanny \& A. R. Khokhlov, Macromolecules 28, 1066 (1995).

6. Q. T. Pham, W. B. Russel, J. C. Thibeault \& W. Lau, Macromolecules 32, 2996 (1999). 